\title{
ANEURISMAS MILIARES DE LEBER
}

\section{LEBER'S MILIARY ANEURYSM}

\author{
BERÁSTEGUI L ${ }^{1}$, ANDONEGUI J ${ }^{1}$
}

\begin{abstract}
RESUMEN
Caso clínico: Varón de 45 años con sensación de escotoma en campo visual derecho. En la imagen del fondo de ojo aparecen dilataciones aneurismáticas con exudación lipídica en arcada temporal superior e inferior. La angiofluoresceingrafía muestra dilatación vascular y extravasación tardía de colorante.

Discusión: La enfermedad de Leber es una vasculopatía retiniana primaria caracterizada por múltiples aneurismas asociados a exudados lipídicos intrarretinianos. El compromiso visual depende de la afectación macular por exudados. El diagnóstico diferencial comprende al resto de vasculopatías retinianas primarias y aneurismas secundarios a otras patologías. El tratamiento es la fotocoagulación con láser argon de los aneurismas.
\end{abstract}

Palabras clave: Aneurismas, Leber, miliar, exudado.

\section{INTRODUCCIÓN}

Los aneurismas miliares de Leber son una vasculopatía retiniana idiopática caracterizada por dilataciones de vénulas y arteriolas que provocan extravasación crónica y exudados duros intrarretinianos. Forman parte de las telangiectasias retinianas primarias junto con las telangiectasias yuxtafoveolares y la enfermedad de Coats. Es importante realizar el diagnóstico diferencial de esta entidad con otras

\begin{abstract}
Case report: A 45-year-old man was referred to us with a scotoma sensation in his right visual field. Funduscopic examination showed aneurysmal dilatations with lipid exudation in the superior and inferior temporal retinal arcades in his right eye. Angiofluorography showed vascular dilatations and late dye leakage.

Discussion: Leber's disease is a primary retinal vasculopathy characterized by several aneurysms associated with intraretinal lipid exudates. Visual compromise depends on macular involvement by the exudates. Differential diagnosis must be established with other primary retinal vasculopathies and secondary aneurysms. Treatment consists of argon laser photocoagulation of the aneurysms (Arch Soc Esp Oftalmol 2008; 83: 669-672).
\end{abstract}

Key words: Aneurysm, Leber, miliary, exudate.

causas de aneurismas retinianos primarios y secundarios ya que el pronóstico visual difiere considerablemente.

\section{CASO CLÍNICO}

Varón de 45 años, sin antecedentes de interés, acude al servicio de urgencias por escotoma en OD de unos días de evolución. La agudeza visual es 9/10

Recibido: 21/5/07. Aceptado: 20/10/08.

Servicio de Oftalmología. Hospital de Navarra. España.

1 Licenciado en Medicina.

Correspondencia:

Lara Berástegui

C/. Avenida de Pamplona, 26, 1. ${ }^{\circ} \mathrm{D}$

31010 Barañain (Navarra)

España

E-mail: gavi_lara@hotmail.com 
en ambos ojos, la presión intraocular es $12 \mathrm{~mm} \mathrm{Hg}$, y la biomicroscopía en ambos ojos es normal. El examen funduscópico del OD muestra cinco dilataciones aneurismáticas rodeadas por exudados lipídicos en arcada temporal superior, y tres en arcada temporal inferior (fig. 1A y 1B). El ojo izquierdo es normal. En la angiografía fluoresceínica (AGF) destaca la dilatación vascular, y la extravasación tardía del colorante (fig. 2A y 2B). Con el posible diagnóstico de aneurismas miliares de Leber, se decide actitud expectante en un primer momento.

A los 4 meses no se aprecia disminución de los exudados por lo que se inicia tratamiento mediante fotocoagulación con láser argon de los aneurismas.

Tras dos sesiones de tratamiento desaparece la exudación. A los cuatro años, en el fondo de ojo, se observan las cicatrices del láser (fig. 3A y 3B).

\section{DISCUSIÓN}

Las telangiectasias retinianas primarias son anomalías de la vascularización retiniana caracterizadas por aneurismas múltiples que provocan extravasación crónica y depósito de exudados duros intrarretinianos. En este grupo se consideran tres entida-

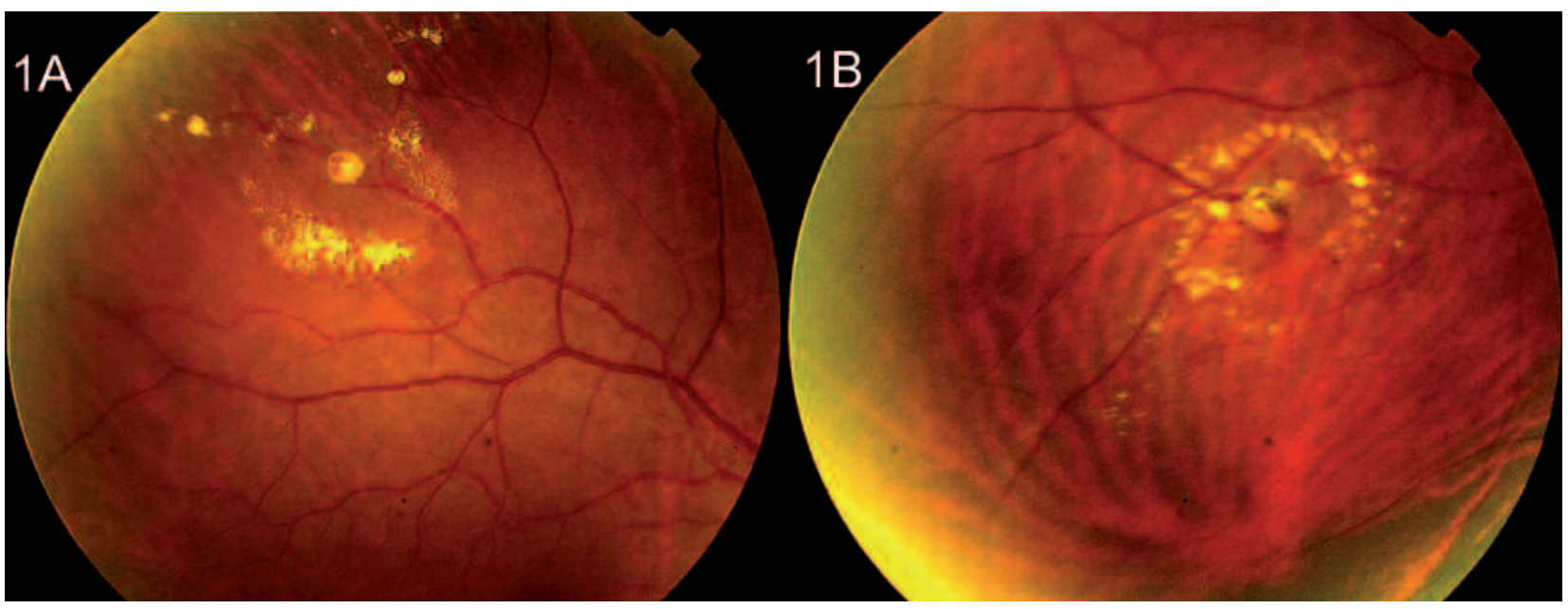

Fig. 1: Imagen del fondo del OD. A: 5 dilataciones aneurismáticas en arcada temporal superior con exudación lipídica alrededor de los mismos. B: 3 aneurismas en arcada temporal inferior.

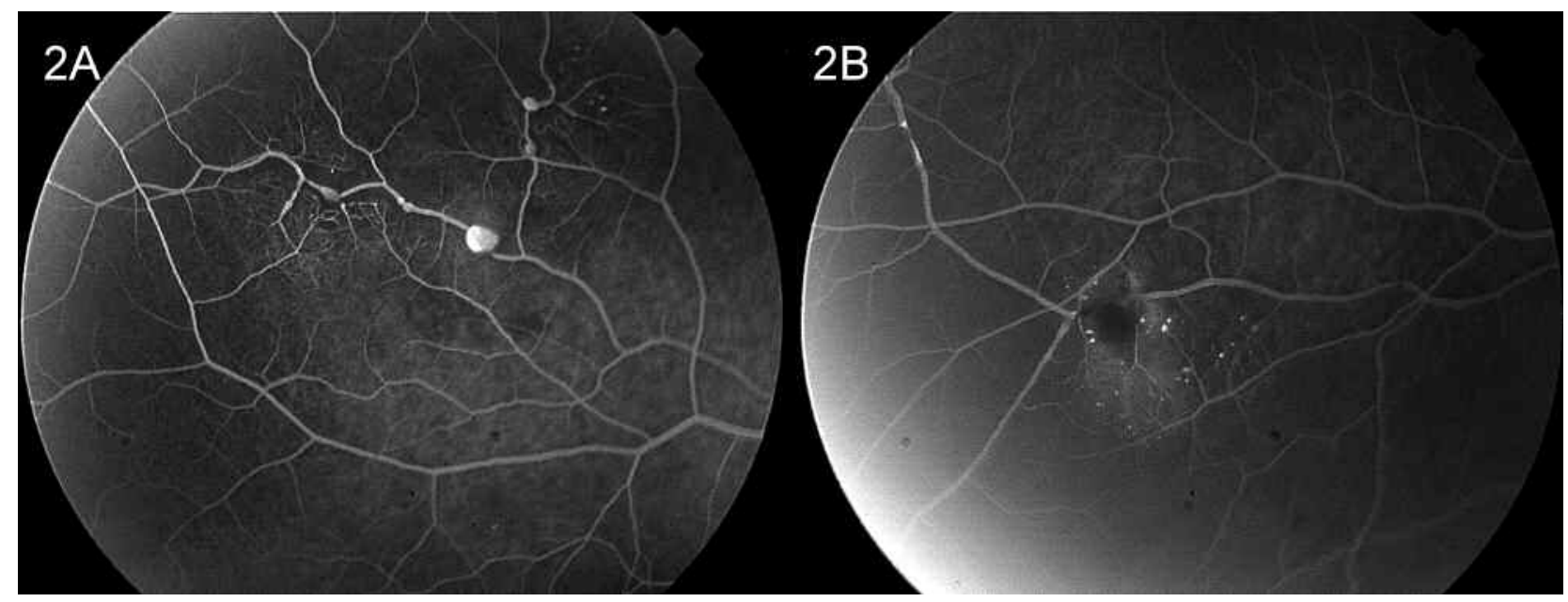

Fig. 2: A: AGF en tiempos precoces (43 sg.) que muestra los aneurismas en arcada temporal superior OD. B: Imagen de arcada temporal inferior (2 min.). 


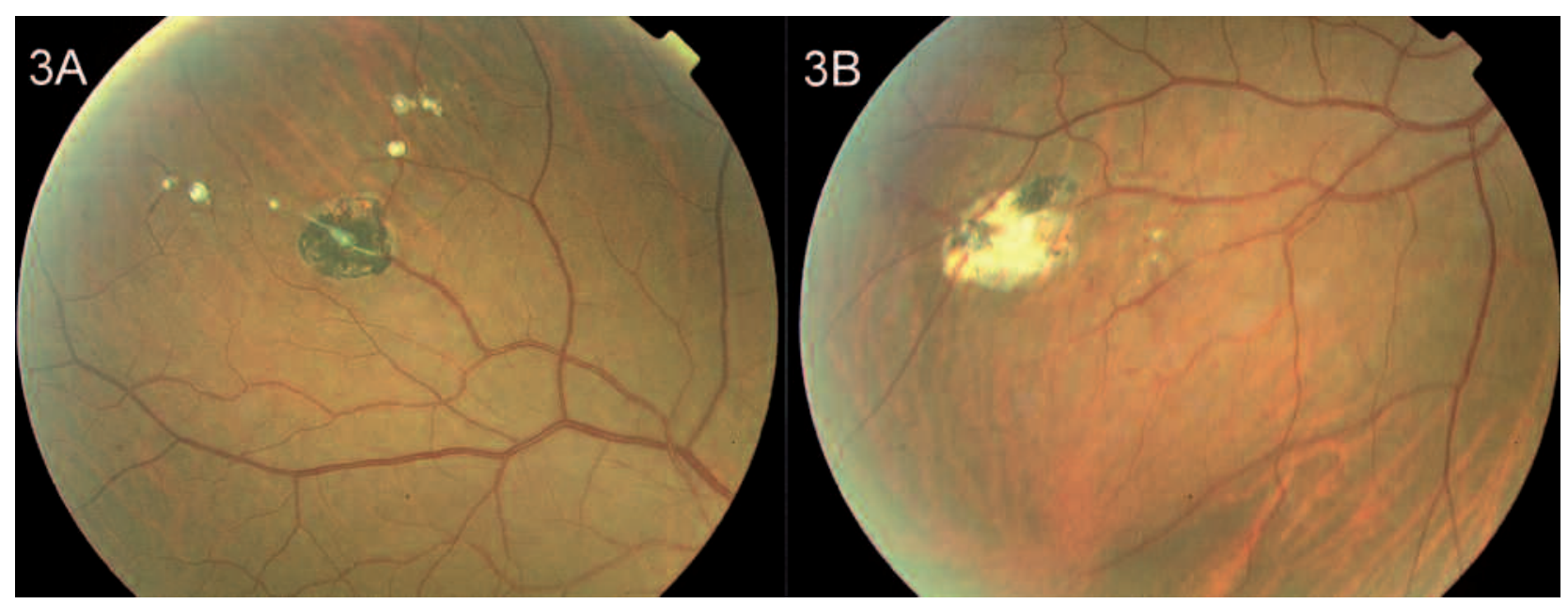

Fig. 3: 4 años después del tratamiento. A: Cicatrices del láser pigmentadas en el lugar en el que estuvieron los aneurismas de arcada temporal superior. B: Imagen de arcada temporal inferior.

des: telangiectasias yuxtafoveolares, aneurismas miliares de Leber y enfermedad de Coats.

Los aneurismas de Leber fueron descritos en 1912. En un primer momento se consideraron una entidad diferente de la enfermedad de Coats. Posteriormente Reese propuso agruparlas como «telangiectasias retinianas primarias» formando parte del espectro de una misma enfermedad.

La enfermedad de Leber se presenta generalmente de forma unilateral, en varones, hacia la cuarta década de la vida. La localización más frecuente es en hemirretina temporal, entre polo posterior y retina periférica.

El diagnóstico se realiza mediante examen funduscópico y angiográfico. Aparecen múltiples aneurismas con extravasación crónica y localizada, que dan lugar a exudados duros. En la AGF, durante la fase precoz, destaca la hiperfluorescencia de las dilataciones vasculares y tardiamente, difusión del contraste desde las telangiectasias pudiendo provocar edema macular cistoide (1).

El diagnóstico diferencial se plantea con otros aneurismas retinianos primarios y secundarios. A diferencia de los aneurismas de Leber, las telangiectasias yuxtafoveolares aparecen en polo posterior (provocando disminución de visión más precoz) y generalmente en mayores de 40 años. El aspecto angiográfico es diferente presentando pequeñas dilataciones vasculares en área perifoveal (2). La enfermedad de Coats es la más grave de las tres vasculopatías primarias. Afecta preferentemente a la edad pediátrica. El compromiso visual es mayor y tienen peor pronóstico porque presentan más exudación y más extensa, pudiendo evolucionar a desprendimiento de retina (3). Se han descrito formas de Coats en adultos con menor repercusión funcional, pero el número de aneurismas y la exudación que provocan es mucho mayor que en los aneurismas de Leber (4). El macroaneurisma arterial retiniano es más frecuente en mujeres, ancianas e hipertensas. Aparecen una o varias dilataciones arteriolares, en una bifurcación o cruce arteriovenoso, y son de localización más posterior. La retinopatía diabética se debe descartar mediante los antecedentes personales o analítica. Los aneurismas son de menor tamaño y se acompañan de otros signos de retinopatía diabética como exudados algodonosos o hemorragias intrarretinianas, que no aparecen en el Leber. En el diagnóstico diferencial se deben añadir otras enfermedades vasculares como oclusión venosa, hemangiomas capilares y cavernosos de retina y descartar la radiación previa como causa de retinopatía con microaneurismas (1).

El caso clínico que describimos se etiquetó de aneurismas miliares de Leber tras descartar enfermedades generales (diabetes, hipertensión, radiación). Se excluyó el Coats porque la exudación era escasa y localizada. La situación lejos de la mácula y la edad hacían poco probable el diagnóstico de telangiectasias yuxtafoveolares.

La progresión de esta entidad suele ser lenta y puede no ser diagnosticada hasta edades avanzadas. El pronóstico depende de la afectación foveolar por los exudados, edema macular, tracción vitreo- 
macular o membrana epirretiniana $(1,5)$. Son posibles otras complicaciones como: trombosis de los aneurismas, neovascularización, hemorragias vítreas y evolución a enfermedad de Coats.

El tratamiento consiste en ablación de los aneurismas mediante fotocoagulación con láser argon si la exudación puede comprometer la mácula. En casos seleccionados se podrían asociar triamcinolona o antiangiogénicos intravítreos y cirugía vitreorretiniana si existe tracción macular o membrana epirretiniana. Si se localizan en periferia se puede plantear crioterapia (1).

En el caso que presentamos se decidió fotocoagular los aneurismas a pesar del carácter focal y la escasa exudación, tras considerar mínima la yatrogenia y para evitar complicaciones posteriores.

\section{BIBLIOGRAFÍA}

1. Bugallo Rivas A, Barbón García JJ, Sanpedro López, A. Telangiectasias retinianas: enfermedad de Leber versus enfermedad de Coats del adulto. Studium Ophthalmologicum 2008; 1: 27-30.

2. Yanuzzi LA, Bardal AM, Freund KB, Chen KJ, Eandi CM, Blodi B. Idiopathic macular telangiectasia. Arch Ophthalmol 2006, 124; 450-460.

3. Shields JA, Shields CA, Honavar SG, Demirci G, Cater J. Clasification and management of Coats disease: the 2000 Proctor Lecture. Am J Ophthalmol 2001; 131: 572-583.

4. Andonegui J, Aranguren M, Berástegui L. Enfermedad de Coats de comienzo en adulto. Arch Soc Esp Oftalmol 2008; 83: 117-120.

5. Sólia Násser L, Paulo de Almeida H, Cabral Zacarias L, Abujamra S, Vieira Gomes AM. Aneurisma miliar de Leber asociado à síndrome de traçao vítreomacular: relato de caso. Arq Bras Oftalmol 2007; 70: 871-874. 